\title{
The application of computer communication technology in the information age
}

\author{
Jia Zhu ${ }^{1, a}$, Chunxue $X u^{1, a}$ \\ ${ }^{1}$ School of Computer Science, Baicheng Normal University \\ a 8zj26@163.com
}

Key words: information age; computer communication technology; security protection

\begin{abstract}
Internet era. Whether it is to buy the occasion effect or the application efficiency has been greatly improved. But it should also be clear that computer communication technology in order to bring great convenience to people's lives, to ensure its safe operation, the maintenance of communication network security has become the biggest risk in the whole technology applications. Once the entire communication system security problems, it will bring immeasurable loss. Therefore, it is very important to develop a perfect security strategy. This article from the current situation of the application of computer communication technology, combined with the existence of security risks, to develop a comprehensive security strategy.
\end{abstract}

\section{Introduction}

With the development of science and technology, themore and more isconvenient Internet technology to provide a great convenience for the transmission of information. However, although the computer communication technology has been widely developed, but it still has many drawbacks, the computer communication technology in order to provide convenience for the development of human society must also make factory, computer technology to further develop and improve, eliminate the existence of security risks and vulnerabilities, security, security of information transmission.

\section{The major advantages of computer communication technology}

Since the computing technology cattle, its technical performance has been the development of more and more excellent quality, since the beginning of twentieth Century the information transfer application is more and more important, the application of computer technology on the development of an important means of information transfer ${ }^{[1]}$. Now, people are still mainly through the use of computer technology to realize the information transmission and communication requirements, of course, with the traditional way of information transmission, computer communication technology has convenient, low cost, safety etc.. In short, the arrival of the information age, whether it is from the national development or from the aspects of social progress, the state will progress to strengthen the construction of computer communication network. The advantages of computer communication technology mainly include the following aspects:

The information transfer more quickly. The main computer communication technology transfer related text, images, sound, video and other information transfer, not only rich in content, also can 
realize the mutual transformation between them, the development of the information age to meet the needs of the great.

The amount of information transmitted. The use of computers for information transmission is the use of digital information technology, this technology in the transmission of information. Under normal circumstances, is not to consider the size of the amount of information. Moreover, using this technology to transmit information, not only to obtain information is very convenient and cheap, we can get a lot of information resources in the network to get a lot of information and do not pay any fees. This huge advantage is mainly because of the Internet digital information transmission mode; this technology will be more widely used in the development of the future ${ }^{[2]}$.

The computer communication technology is more secure. Information tool is for using computer technology transmitting. With the advent of the information age, the security of information and personal privacy has attracted more and more attention. In the design of the Internet software will encrypt the information, even the professionals are very difficult to crack at the same time, with the development of programming techniques and a variety of anti-virus software encryption software for the strengthening of emerge in an endless stream, a guarantee of information security. The use of computer technology for the transmission of information is not only fast, large capacity, low cost, and high reliability, the development of computer technology, information technology is necessary to carry out the strong trend ${ }^{[3]}$.

\section{Application of computer communication technology}

The application of computer communication technology mainly includes the following aspects: Internet technology is about the computer communication technology. Making the application of computer communication technology continues to expand. At present, the country has been expanding the construction of optical cable systems, as well as the development of Internet technology, both for the development of the communication technology to provide convenient. Moreover, in the continuous development of the performance of computer communication technology will also be further improved, a variety of software systems at the same time now emerging made the function of the computer will be more complete an application in all walks of life. All of these are the important driving force for the wide use of computer technology, and with the continuous improvement of the infrastructure, the computer communication technology combined with the Internet will have a more rapid development.

Computer technology is to achieve the application of Multimedia. With the popularity of computer hardware, the computer is used as the carrier of all kinds of communication software. At the same time, the emergence of smart phones, tablet PCs and so on also provides convenience for the wide use of this software ${ }^{[4]}$. At the same time, with the continuous development of computer hardware, the multimedia communication technology will be further developed and perfected, it is more important to this form of communication is convenient, rich and the most reliable, simple and safe operation, all these will make the multimedia communication technology has been widely applied. And in the future in the form of Multimedia Office, communication, information transmission will be widely used, provide convenience for people's work and life, make life more agile, never easy and efficient access to information, more convenient, more perfect social development.

Computer technologies enable the realization of remote communication. The use of computer technology to achieve communication is not a simple process; first of all, it must be connected to the Internet so that computers can communicate with other computers around the world for 
information exchange and communication. Furthermore, to achieve the function of the software carrier, the exchange of information will be two computers connected together, this technology allows people around the world can also be carried out anytime, anywhere communication. At the same time, in the use of the Internet search for the necessary information, the computer will enter the information through the keyboard to screen thousands of information, convenient and quick.

\section{Computer communication technology information security method}

With all walks of life to all who rely on computer technology more and more strong, the reliability and safety of the use of computer information exchange becomes more and more important to people at present, mainly through the following means to strengthen the security of computer information.

The Internet technology is development. With the development of Internet technology QQ, We Chat and other chat tools are widely used in the factory, if the information delivery system encryption technology is still not strong enough, it is likely to be personal information leaked to others. For the transmission of information security, we strengthen the encryption transmission system information at the same time, and strengthen the construction and improvement of the firewall, additional antivirus software on time to clean up the hard disk of the computer, ensure the computer performance is safe and reliable. Secondly, for the criminals to steal others' information factory discipline, the state should regulate the behavior of the development of relevant laws, and strengthen the supervision of the network communications market, a day appeared to steal others' information of wrongdoing severely hit them, serious when can also according to the law of criminal punishment in order to ensure the security of the computer system. Secure. Finally, strengthen account security management; firewall in general, user account has a relatively wide cover, in the process of account login system, it is necessary to ensure a valid account number and the correct password. The process of direct attack network system, is often to steal account and password, and the user account in strengthening the process of safety management, to set up the complex passwords, as far as possible on a different account and password settings, a combination of letters and numbers and symbols of the account password based settings, and change your password regularly.

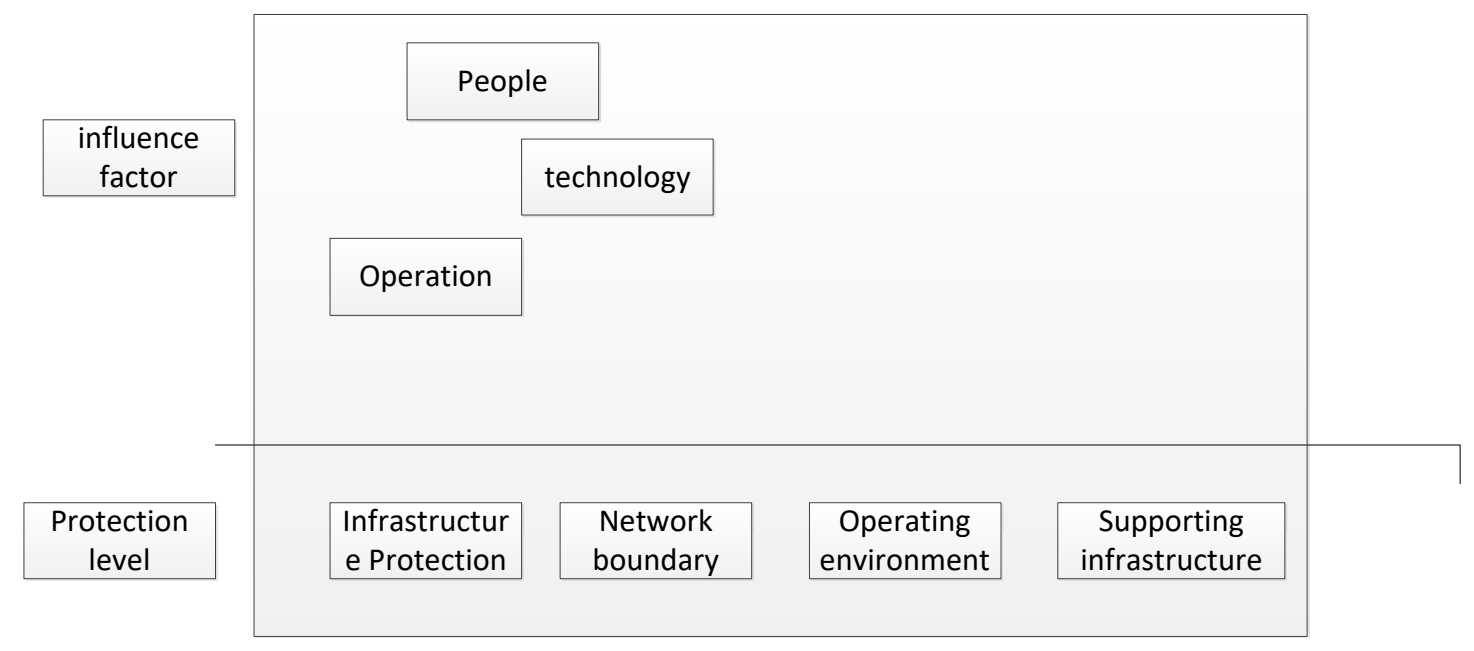

Fig1. Schematic diagram of deep defense system

Network firewall technology is present. This is mainly to the access control network between the overall strengthening of the external network; avoid the illegal means to control the process, effective access to good internal cyber source, network interconnection devices on the internal 
network operating environment protection. Transmission of data packets between multiple networks, combined with the related security strategy, reasonable to ensure that good communication equipment network, close monitoring of the network running status are different, combined with the technical characteristics of the firewall, the firewall exists not only packet filtering type and agent type, there is also a type of monitoring. A packet filtering firewall, application of packet transmission technology, after the data packet read information, judge of trust on information, once dangerous according to the package, the process will do reject. For the address type of a firewall control process, combined with the inside of the IP address, the temporary IP address to the conversion, in the internal network access control phase, the real 11'address hidden. A day is access to the internal network, the lack of internal network connectivity solutions direct factory. in the process of external network For the proxy type of Firewall installation process, mainly to achieve the data request, and combined with the server to obtain data, and then realize the data transmission. This external system is application process, to avoid the external direct damage to the internal network system. For monitoring firewall installed, often illegal intrusion on the site at all levels of the virus a judgment, use of the distributed detector to judge the node information, has a strong role in prevention and control. And the personal use of the computer process, the firewall is not only to do a good job of software firewall control, but also to combine anti-virus software, to achieve the process of supporting the installation. For a variety of security technology, the use of the process, to avoid a defense against the Trojans, and the combination of anti-virus software in a timely manner to upgrade the latest version of the upgrade, do a good job of anti-virus.

\section{Conclusion}

In a word, with the development of information technology in the era of the application will be more and more perfect and mature, now people have been able to realize through the communication of information technology so that whenever and wherever possible, relatives and friends in different places to lift the pain. Moreover, in recent years, more and more extensive online shopping, greatly enrich people's right, and thus promote the development of the financial industry. It is because of the convenience of Internet technology, the people's daily life more and more dependent on the Internet, criminal's also fancy factory Internet operation implied huge benefits at the expense of the chain, by way of profiteering, the safe operation of the Internet is threatened. The development and use of any new things are two sides of the Internet too, but the overall application of Internet technology is still very good, we should further improve the Internet technology to make it better for people.

\section{References}

[1]The development and application of computer communication technology[J].communication world, 2013 (16).

[2] Chen Li Jun.on the application of computer communication technology in the information age [J] Silicon Valley, 2014 (01).

[3]Lu Xiumei. Research on the development and application of computer communication technology [J]. information system engineering. 2015 (11).

[4]Chen Long. The development trend of computer communication technology to explore [J]. China new communication. 2014 (20). 